\title{
Die Anfänge der Konsistorialverfassung im lutherischen Deutschland.
}

\author{
Von \\ Karl Müller in Tübingen. ${ }^{1}$ )
}

Uber die Anfänge der Konsistorialverfassung herrscht heute im wesentlichen Ubereinstimmung. Die Konsistorien, so sagt man, treten nach ihrer ursprünglichen Bestimmung, wie sie in den kursächsischen Anfängen vorliegt, an die Stelle der Bischöfe und der bischöflichen Behörden; ihr Wirkungskreis umfaßt die Jurisdiktion im kanonischen Sinn, also Gerichtsbarkeit und Verwaltung. Sie ersetzen also die früheren unständigen Visitationskommissionen, mit denen die Reformation begonnen hatte, sie müssen deshalb als Organe des gesamten landesherrlichen Kirchenregiments betrachtet werden. ${ }^{2}$ ) Daneben wird wohl be-

1) Ich veröffentliche hiermit den Vortrag, den ich auf dem Internationalen Kongreß für historische Wissenschaften zu Berlin im August 1908 gehalten habe, und füge nur die wichtigsten Belegstellen bei. Auf den Gedanken, das Thema in größerem Umfang zu behandeln, mußte ich wegen anderweitiger Aufgaben verzichten.

2) Vgl. vor allem K. Rieker, Die rechtliche Stellung der evangelischen Kirche Deutschlands S. $161 \mathrm{u}, 164 \mathrm{ff}$. (speziell von den Wittenberger Entwürfen von 1538 u. 1542). S. 175 M. - Die Konsistorien als Ersatz für die unständigen Visitationskommissionen: Rieker S. 174 f. nach O. Mejer, Das Rechtsleben der deutschen evangelischen Landeskirchen S. 26. Sehling in der

Historische Zeitschrift (102. Bd.) 3. Folge 6. Bd. 
merkt, daß die Konsistorien in der Praxis nicht überall gleich gestaltet gewesen seien, indem sie zunächst bald nur die eigentliche Jurisdiktion, bald die Verwaltung ohne Gerichtsbarkeit, bald die volle Verwaltung der Kirche, also die Jurisdiktion im kanonischen Sinn, umfaßt hätten. ${ }^{1}$ ) Allein man macht davon in der Darstellung der Geschichte der Konsistorien kaum irgend welchen Gebrauch, und in dem bedeutsamen und viel genannten Werk von K. Rieker ist dieser Punkt geradezu wieder ausgeschieden. ${ }^{2}$ )

Ich möchte diesem Punkt näher nachgehen und zeigen, daß von Haus aus zwei verschiedene Typen von kirchlichen Behörden vorliegen, die von ganz verschiedenen Punkten aus konstruiert und erst allmählich kombiniert oder einander angenähert worden sind. Freilich kann ich nur einen vorläufigen Versuch bieten. Denn wohl ist durch Sehlings Kirchenordnungen das Material bedeutend vermehrt worden, aber vorerst doch nur für die beiden sächsischen Fürstentümer und ihre nächste Umgebung und auch da fast nur für die Gesetzgebung. Für die Praxis der Behörden sind wir nach wie vor sehr arm an Material. Und doch kann sie allein für viele Punkte Klarheit und Anschaulichkeit bringen.

Realencyklopädie für protestantische Theologie und Kirche ${ }^{\mathbf{3}} 10$, $755_{20}$ f.

1) L. A. Richter, Geschichte der evangelischen Kirchenverfassung S. 120. Ahnlich O. Mejer a. a. O. S. 27. Sehling a. a. O. S. $755_{52}$ ff. - Anders K. J. Stahl, Die Kirchenverfassung nach Lehre und Recht der Protestanten ${ }^{2}$ 1862, S. 306 ff. Aber seine Aufstellungen haben sehr begreiflichen Widerspruch hervorgerufen. Auch R. Sohms Darstellung in seinem Kirchenrecht 1, 609-630 hat solchen reichlich gefunden (z. B. Rieker S. 173 f.); aber seine Ausführungen im einzelnen sind damit nicht widerlegt. Sie enthalten vielmehr sehr bedeutsame Elemente, die $\mathrm{m}$. E. lange nicht genug gewürdigt sind, hier aber nicht verfolgt werden können.

2) Ich verweise auf seine Ausführungen S. 174 f., bes. S. $175 \mathrm{~d}$ Abs. 2, wo ganz allgemein auf die Bedeutung des Umstandes hingewiesen ist, da $\beta$ damals Verwaltung und Rechtsprechung nicht geschieden gewesen seien und daher die Bezeichnung der Konsistorien als „Kirchengerichte“ über ihren Charakter nichts aussage. 
Die Anfänge der Konsistorialverfassung im luther. Deutschland. 3

I.

In allen fürstlichen Territorien Deutschlands war die Reform der bestehenden Zustände durch Visitationen eingeleitet worden, und durch das ganze 16. Jahrhundert hindurch wiederholen sie sich. Sie werden von Kommissionen vorgenommen, die, vom Landesherrn ernannt, aus Theologen, fürstlichen Räten, Adligen und u. U. auch Vertretern des städtischen Bürgertums bestehen. Aber sie werden, wie das ja damals noch vielfach auch im Gebiet der weltlichen Verwaltung geschieht, immer nur von Zeit zu Zeit und meist nur für einzelne Gebiete zusammenberufen. In der $Z$ wischenzeit besteht zunächst keine andere Instanz für das kirchliche Regiment des Landesherrn, als dieser selbst und seine Räte, die Kanzlei, Ratsstube oder wie sonst der Name sein mag.

Zuerst in Kursachsen hat man $1527 \mathrm{f}$. ständige Beamte geschaffen in den Superintendenten. Ihr mittelalterliches Vorbild ist freilich nicht der Bischof - dazu ist schon ihr Bezirk viel zu klein; sie sitzen in jeder Amtsstadt - , sondern der richterliche Beamte des Bischofs, der Offizial, Kommissar, auch Landdechant. Der Superintendent ist an der Ehegerichtsbarkeit wenigstens mit den weltlichen Amtleuten und anderen Personen zusammen beteiligt, prüft die Geistlichen seines Bezirks vor ihrer Anstellung und übt die Aufsicht über sie, indem er nach kanonischem Vorbild auf clamor und fama publica oder auf denuntiatio hin einschreitet ${ }^{1}$ ) und

1) Die herrschende Anschauung überschätzt m. E. erheblich die Aufsichtspflichten, die den Superintendenten am Anfang übertragen worden sind, und die eingehende Darstellung von $\mathrm{H}$. Nobbe, Das Superintendentenamt, seine Stellung und Aufgabe nach den evangelischen Kirchenordnungen des 16. Jahrhunderts in der ZKG. 14, $404 \mathrm{ff}$., $556 \mathrm{ff}$. und 15, $44 \mathrm{ff}$. erweckt schon darum falsche Vorstellungen, weil sie viel $z u$ wenig die Entwicklung des Amtes ins Auge faßt. - Auszugehen ist von der kurfürstlichen Instruktion für die Visitatoren von 1527 und dem Unterricht der Visitatoren von 1528. In der Instruktion (Richter, KOO. 1, 80 b u. d. M.; Sehling, KOO. I, 1, 146 a u.) wird den Superintendenten befohlen, Aufsicht darüber zu führen, ob die Geistlichen ihres Bezirks den Vorschriften der Instruktion nachleben. „Und so der 
im Notfall die Sache an den Amtmann oder Kurfürsten weitergibt. Denn eines fehlt ihm, was dem kirchlichen

superintendens... befunde oder an inen gelangen wurde“, $\mathrm{da} \beta$ ein Geistlicher falsch lehre, mit den Sakramenten und Zeremonien anders handele oder einen bösen Wandel führe, solle er ihn zu sich fordern, ihm die Unschicklichkeiten, die a $\mathrm{n}$ ih $\mathrm{n}$ gelangt, vorhalten, seine Verantwortung anhören und, wenn er leugnen sollte, sich weiter erkundigen und die Sache an den Kurfürsten bringen. - Deutlicher noch im Unter richt (Richter 1, 99 a u.; Sehling I, 1, 171 a u.): „Wo nu der [Lehre, Zeremonien, Leben] eins odder mehr von einem odder mehr pfarrherrn oder predigern vernomen oder gehandelt würde" (usw. wie in der Instruktion). Dazu die Kirchenordnung für BraunschweigWolfenbüttel 1543 (Richter 2,58a): „Und wenn en [den Super-

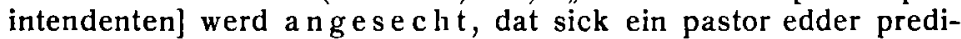
cante nicht vlitich holt in der ler und in dieser ordeninge edder övel lebet" usw. Endlich die albertinisch-sächsischen Generalartikel von 1557 (Richter 2, 182 a u.; Sehling I, 1, 321 a ï. d. M.): ${ }_{n} \mathrm{Da}$ auch der superattendens etwas ungebürlichs oder streflichs von einigem piarherr... selbst erfaren oder aber vom lehenherrn oder den eingepfarten e rku ndigung be k ommen hette" usw.

Offenbar sind hier zumeist die zwei Fälle unterschieden, daB der Superintendent etwas durch Hörensagen oder durch Anzeige erführe. Das „befunde“ der Instruktion setzt auch keine Visitation in der Pfarrei selbst voraus. Vgl. die Sächsischen Generalartikel von 1557 (Richter 2, $182 \mathrm{a}$ M. Sehling I, 1, $320 \mathrm{~b}$ M.), die in einer Zeit viel entwickelterer Aufsicht vorschreiben: der Superintendent solle seine Aufsicht ausüben, indem er 1. die Geistlichen zu sich (zur Synode) bescheide, 2. wenn es die Notdurft erfordere, unangemeldet in die Gemeinden gehe. Dieser zweite Modus wird dann nachher so wieder aufgenommen: „Und wo also, wie obberürt, einer oder mehr superattendenten etzlicher irer kirchen gelegenheit dermaßen befunden, daB die zu besuchen von nöten", usw. Hier ist ja deutlich, daB die Visitation an Ort und Stelle erst dem "Befund“ folgt. Der Befund kann also nicht selbst eine Visitation voraussetzen. Aber auch mit der Besprechung auf den Synoden besteht kein Zusammenhang. Offenbar setzt also auch hier das „befinden“ nur Anzeige oder Gerücht voraus. In dem Entwurf der Wittenberger Konsistorialordnung von 1542 (Richter 1, 370 a oben und Sehling I, 1, 203 a u. d. M.) heißt es: „und wo einer... befunden oder berïchtiget" usw.

Darin aber sind beide Voraussetzungen gegeben, unter denen nach dem kanonischen Recht der geistliche Vorgesetzte gegen seine Untergebenen einschreitet: a) fama publica, clamor, clamosa insinuatio, b) denuntiatio. Vgl. meinen Aufsatz über Luthers römischen Prozeß in der ZKG. 24, $54 \mathrm{f}$. 
Die Anfänge der Konsistorialverfassung im luther. Deutschland. 5

Richter der Vergangenheit wesentlich gewesen war, die selbständige Gerichts- und Strafgewalt.

Allein bald erschien dieses eine Amt als ungenügend. Vor allem entwickelten sich auf dem Gebiet der Ehegerichtsbarkeit schwere Notstände. Weder die Amtleute noch die Superintendenten waren dafür vorgebildet. Das bisherige Eherecht war durch Luther zum Teil umgestürzt, und ein neues, das den evangelischen Grundsätzen entsprochen hätte, bestand noch nicht. Eine gleichförmige Praxis aber konnte sich so lange nicht bilden, als die zahlreichen Ehegerichte der einzelnen Amtsbezirke zusammenhangslos nebeneinander bestanden. So verlangte man nach wenigen, aber weitreichenden Ehegerichten, die, aus Sachverständigen, also Theologen und Juristen, bestehend, einheitliche Grundsätze und gleichförmige Praxis für das ganze Land schaffen könnten.

Dazu kamen die wachsende Auflösung der Sitte in den Gemeinden und die unbefriedigenden Verhältnisse unter dem geistlichen Personal. Da schienen die seelsorgerlichen Mittel nicht mehr auszureichen, und wenn Superintendenten oder Visitatoren die weltliche Hilfe der Amtleute dagegen in Anspruch nahmen, so wurde sie ihnen nicht oder nur ungenügend gewährt. So schien der einzige Ausweg, daß man geistliche Zuchtgerichte schüfe, die mit dem Recht, $Z$ wangsstrafen zu verhängen, und mit eigener Exekutive ausgestattet wären.

Das waren die Gründe, die den großen landschaftlichen Ausschuß 1537 veranlaßten, den Kurfürsten um Errichtung von Konsistorien zu bitten, die an die Stelle der zahlreichen bischöflichen Richter der früheren Zeit treten und als Ehe- und Zuchtgerichte mit selbständiger Gerichts- und Strafgewalt wie mit eigener Exekutive ausgestattet sein sollten. Ein Gutachten von Wittenberger Professoren, vor allem Justus Jonas, aus dem Jahr 1538 veranlaßte den Kurfürsten, 1539 wenigstens e in Konsistorium zu Wittenberg für den Kurkreis und den Kreis Torgau zu errichten. Allein es bekam keine feste Bestallung und arbeitete zunächst auch nur als Ehegericht. Erst 1542 erhielt es den Auftrag, den Entwurf 
für eine endgültige Konstitution auszuarbeiten. In diesem Entwurf ist das Gutachten von 1538 fast ganz verarbeitet, aber namentlich ein Abschnitt über Visitation und Inquisition hinzugefügt worden. ${ }^{1}$ )

Das Gutachten wie der Entwurf weisen nun in ihren Motiven lediglich auf einen Gerich tshof, der über die Ehesachen, die geistlichen Personen und die kirchlichen Vergehen der Laien richten soll. Die Konsistorien sind also, dem bisherigen Gebrauch des Namens gemäß, Kirchengerichte und heißen auch in Zukunft häufig wirklich so.

Nun ist es ja ganz richtig, daß das $k$ anonis che Recht keine Trennung von Rechtsprechung und Verwaltung kennt. Aber man kann daraus nicht schließen, daß die Konsistorien von vornherein beides, also das gesamte Kirchenregiment, vereinigen, den vollen Ersatz für die bischöfliche Gewalt hätten bilden sollen. Einmal waren sie jetzt landesherrliche Behörden, und in den fürstlichen Territorien der Zeit ist die Trennung der zentralen gerichtlichen und der Verwaltungsbehörden in erheblichem Umiang begonnen oder durchgeführt. Sodann aber auch die causae ecclesiasticae, die den Konsistorien zufallen sollen, sind immer wieder unmißverständlich so beschrieben, daß darunter eben jene drei Punkte fallen, Ehesachen, Lebens- und Amtsführung der Geistlichen und kirchliche Vergehen der Laien. ${ }^{2}$ ) Wenn aber dabei die Aufsicht über Geistliche und Gemeinden, ja auch einigermaßen - Freilich nur in sehr beschränktem Maß über Kirchen, Kirchhöfe, Güter und Häuser der Pfarreien eine Rolle spielt, so verläuft sie eben in den Formen des Gerichts, der inquisitio, nämlich entweder als Einschreiten im einzelnen Falle, offenbar wieder auf clamor oder

1) Das Gutachten s. bei Richter, Geschichte der evangelischen Kirchenverfassung S. 82 ff., den En tw u rf bei Richter, Kirchenordnungen 1, 367 ff., Sehling I, 1, $200 \mathrm{ff}$. Dazu vor allem O. Mejer, Zum Kirchenrecht des Reformationsjahrhunderts: Die Anfänge des Wittenberger Konsistoriums S. $45 \mathrm{ff}$.

9) Vgl. das Gutachten besonders S. 83 5-7. 23, 84 5. 6. 11, 8425 bis $8540,8625 \mathrm{ff}$., $9517 \mathrm{f}$. 
denuntiatio hin, oder als generelles Verfahren im Wege der Visitation, des Sendgerichts, das hier selbst mit seinen technischen Ausdrücken aufgenommen wird. ${ }^{1}$ )

So erscheint auch die Aufsicht über das Vermögen der Pfarreien sowie die "Defension" der Geistlichen in dieser Form. Das Konsistorium ist der Ort, da die Geistlichen klagend ihr Recht gegen alle Beeinträchtigungen sollen suchen können. ${ }^{2}$ ) Die Aufsicht über den Stand der Pfarrgüter aber wird gefaßt als ein Stück der

1) Das Gutachten spricht nur davon, daß die Kommissare nach den verschiedenen Richtungen bei Geistlichen und Gemeinen ein Ein- oder Aufsehen haben, Achtung geben, Nachirage tun sollen; es denkt dabei offenbar an die einzelnen Fälle, da Gerücht oder Anzeige das Einschreiten veranlassen. Der Entw urf fügt das generelle Verfahren im Wege des Sendgerichts hinzu u. d. T.: Von der Visitation und Inquisition (Richter, KOO. I, $371 \mathrm{ff}$., Sehling I, $204 \mathrm{~b}$ ff.). Die kanonistisch-rechtlichen Ausdrücke (s. Richter S. 371 b oben, Sehling S. 205 a ii. d. M.) weisen, wie auch längst und allgemein erkannt ist, deutlich auf das Sendgerichtsverfahren: darauf weisen die Personen, die zur "Visitation“ erfordert sind und vom Kommissar auf ihre Pflicht hin ausgefragt und zur Anzeige und zum Bericht aufgefordert werden, der Notar, der ihre Aussagen aufzeichnet, der Kommissar, der dann ex officio gegen die Diffamierten prozediert, der ${ }_{\text {Bericht }}$, der als denuntiatio canonica generalis et publica de peccato corrigendo der alten Zeit gelten soll, die purgatio, die dem Leugnenden auferlegt wird. Sarcers Mansfelder Visitationsordnung von 1554 (Richter 2, 143 b oben, Sehling I, 2, 192 a unten) spricht denn auch von den Sendschöppen, die vor Zeiten lateinisch Scabini synodales genannt worden seien. Dazu vgl. Sarcer, Von den Mitteln und Wegen, die rechte und wahre Religion $z u$ befördern und zu erhalten 1555 fol. 308 ; auch 311 b, wo er von den Decanis ruralibus oder "Senddechanten" spricht, wie man sie vor Zeiten genannt habe. (Uber diese Schrift vgl. besonders $O$. Mejer, Die Grundlagen des lutherischen Kirchenregiments S. $124 \mathrm{ff}$.) Vgl. auch die Xußerung Georgs von Anhalt bei Sehling, Kirchengesetzgebung unter Moritz von Sachsen S. 30: „Die Institution der Rüge, wie sie früher auf den Dörfern gehalten worden sei - daß nach den Gebrechen gefragt worden sei $^{6}-$, sei eine gute Einrichtung gewesen." Das ist eben das Sendgericht.

2) Vgl. das Gutachten S. $88_{40-898}$, den Entwurf bei Richter 1, 370a Abs. 2, Sehling I, 1, 203 a unten. Dasselbe findet sich z. B. in dem Gutachten der Räte des Herzogs Moritz bei E. Brandenburg in der Historischen Vierteljahrschrift 4, 213: solche Klagen (gegen den Adel) sollen an das Konsistorium gehen. 
Aufsicht über die Amtstätigkeit der Geistlichen, nämlich ob sie das ihnen zur Nutznießung überlassene Pfarrgut nicht herunterwirtschaften ${ }^{1}$ ); und die Aufsicht über die Kirchen, Kirchhöfe und kirchlichen Gebäude erscheint als eine solche über die christlichen Tugenden der Gemeinden und Patrone, ob sie ihre Pflicht gegen jene Immobilien erfüllen. ${ }^{2}$ )

So sind also die Konsistorien ganz vom Gedanken der Kirchengerichte aus begründet und entworfen.

Indessen ist der Entwurf von 1542 auch nicht ausgeführt worden. Es blieb bei dem einen Wittenberger Konsistorium, und das diente nur als einfaches Ehe- und Zuchtgericht. Und als Zuchtgericht hatte es nur unbedeutende Kompetenz: aus dem Sendgericht wurde nichts, und eigene Exekutive bekam es auch nicht. ${ }^{3}$ )

Die zweite Etappe in der Geschichte der Konsistorien liegt im albertinischen Sachsen. Hier wird seit 1544 über die künftige Verfassung verhandelt. Ich hebe nur e ine Episode heraus.

Eine Leipziger Theologenkonferenz von Lätare 1544 wünscht ein Konsistorium ganz nach dem Muster des Wittenberger Entwurfs von 1542 als Gerichtshof und Sendgericht für das ganze Land. Sie möchte ihm aber auch Prüfung, Ordination und Institution der neu berufenen, noch nicht anderweit - etwa von der Wittenberger Fakultät - ordinierten Prediger sowie die Prüfung und Bestätigung der Superintendenten übertragen. Sie möchte ferner - und das ist ein Ziel, das nun in Sachsen wie in anderen Territorien immer deutlicher hervortritt - die allgemeine kirchliche Aufsicht erweitern. Ihr Ideal wären regelmäßige jährliche Visitationen der

1) Vgl. den Entwuri bei Richter 1, 372 a Abs. 3. Sehling I, 1, 205 b M. (Nr. 5).

2) Gutachten S. $91_{3}$ ff. Entwurf bei Richter S. 370 b oben. Sehling S. $203 \mathrm{~b}$ unten.

$\left.{ }^{3}\right)$ Vgl. O. Mejer, Zum Kirchenrecht des Reformationsjahrhunderts: Die Anfänge des Wittenberger Konsistoriums S. 56 ff. 
Die Anfänge der Konsistorialverfassung im luther. Deutschland. 9

Superintendenten in allen Pfarreien. Aber das erscheint ihr zunächst undurchführbar. Darum soll der Superintendent ${ }^{1}$ ) wenigstens jährlich seine Geistlichen zur Synode vor sich bescheiden, sich über ihr Leben und Lehren erkundigen und die einzelne Gemeinde besuchen, wenn er von dort besondere Notstände erfahren hat. Außerdem aber soll jedem Superintendenten ein Kirchengericht für seinen Bezirk beigegeben werden, das an der Aufsicht über die Verwaltung der Gemeinen Kasten beteiligt sein, vor allem aber mit dem Superintendenten zusammen ein Zuchtgericht für den Bezirk, jedoch mit bloßer Mahngewalt, bilden soll. Das Bedürfnis nach verstärkter Aufsicht zieht somit auch die Erweiterung und Abstufung der Kirchengerichte nach sich. ${ }^{2}$ )

Allein diese Vorschläge der Theologen wurden nicht ausgeführt. Es kam vielmehr die bischöfliche Episode der sächsischen Kirchenverfassung unter Fürst Georg von Anhalt als Bischof oder Koadjutor von Merseburg. In diesem Stadium erscheint das Konsistorium als Behörde des Bischofs.

Während nun aber diese Verfassung nach dem Schmalkaldischen Krieg mit dem erzwungenen Rücktritt des Fürsten Georg zusammenbrach und nach dem Passauer Vertrag das la nde sherrlich e Kirchenregiment in seinem ganzen Umfang eintrat, blieb doch ein Punkt aus der Verwaltung des Fürsten Georg bestehen: die verschärfte Aufsicht über Geistliche und Gemeinden. Zwar hatte Herzog Moritz schon 1546 den idealen Wunsch der

1) Dabei wird ein Gedanke des Wittenberger Entwurfs von 1542 benutzt (Richter S. 371; Sehling S. 204 b und 205 a). Uberhaupt schließen sich die Verfasser stark an den Entwurf an, wie schon Sehling, Kirchengesetzgebung S. 2 bemerkt hat.

2) Die Vorschläge der Leipziger Lätarekonferenz sind von Sehling, Kirchengesetzgebung unter Moritz von Sachsen S. $121 \mathrm{ff}$. veröffentlicht und verwertet. Dann hat E. Brandenburg auch uber sie gehandelt in seinem Aufsatz: Entstehung des landesherrlichen Kirchenregiments im albertinischen Sachsen, Histor. Vierteljahrschrift 4, 195 ff. Ich setze mich mit beiden in dem Exkurs am Schlusse auseinander, beschränke mich jedoch auf das, was unmittelbar mit diesem Vortrag zusammenhängt. 
Theologenkonierenz, daß die Superintendenten alle Pfarreien an Ort und Stelle visitieren möchten, mit Rücksicht auf den Adel abgelehnt. ${ }^{1}$ ) Die Lokalvisitation blieb ein Mittel für außerordentliche Notstände, und auch aus dem Sendgericht der Konsistorien ist nichts geworden. Dagegen waren die regelmäßigen jährlichen Synoden der Superintendenten mit ihren Geistlichen bewilligt und vom Fürsten Georg meisterhaft ausgeführt worden. ${ }^{2}$ ) So wurden sie denn auch in die neuen Verhältnisse hinübergenommen. Die drei Konsistorien aber, die Kurfürst August nunmehr errichtete, waren wieder fast nur Gerichtshöfe. $\left.{ }^{3}\right)$ Weitergehende Vorschläge, ihnen z. B. den Vollzug der Ordination und einen regelmäßigen Anteil an der Aufsicht über einzelne Teile der Verwaltung des Gemeindevermögens $z u$ übertragen, sind abgelehnt worden. ${ }^{4}$ )

Auch in den übrigen mittel- und norddeutschen Territorien, die in dieser Zeit Konsistorien bekamen, sind diese Behörden nur oder wenigstens durchaus in erster Linie Gerichtshöfe im wesentlichen in dem Umfang, wie wir ihn bisher kennen gelernt haben. Immerhin ist zu bemerken, daß die Konsistorien jetzt zum Teil schon in ihrer grundlegenden Organisation auch weitere Auf-

1) Vgl. das Bedenken der Räte Herzog Moritzens in der Hist. Vierteljahrschrift 4, 231 und dazu die Bemerkung Brandenburgs S. $219 \mathrm{M}$.

z) Uber seine Praxis s. z. B. das Gutachten des Leipziger Konsistoriums von 1556 , das Sehling veröffentlicht hat in der Deutschen Zeitschrift für Kirchenrecht (3. Folge) 13, $210 \mathrm{ff}$.

$\left.{ }^{8}\right)$ Sehling, Kirchenordnungen I, 1, $340 \mathrm{f}$.

4) Die Vorschläge für die Ordination s. in dem Gutachten des Leipziger Konsistoriums von 1556, DZKR. 13, 210 ff. Für das kirchliche Vermögen vgl. bei Sehling I, 1, 316 a oben die Anm. I und 3, wo die abweichenden Lesarten der Magdeburger Handschrift verzeichnet sind. Dazu die Bemerkung Sehlings I, 1, 109, der das Magdeburger Exemplar als einen Entwurf für die Generalartikel von 1557 ansieht. Jedenfalls sind jene Bestimmungen ïber den Anteil des Konsistoriums weder in der Visitationsordnung von 1555 noch in den Generalartikeln von $1557 \mathrm{zu}$ finden. - DaB die Konsistorien Gutachten abzugeben haben (Sehling S. 106 u. 110 Abs. 5 u. 7), ist ja etwas ganz anderes. Eigentümlicherweise ist die Prüfung und Konfirmation der Dorfkiister den Superintendenten oder dem Konsistorium übertragen (S.326 a M.). 
Die Anfänge der Konsistorialverfassung im luther. Deutschland. 11

gaben übertragen bekommen. Es war ja nur natürlich, daß, wenn man einmal eine solche spezifisch kirchliche Behörde hatte, man ihr mehr und mehr auch andere als eigentlich gerichtliche Aufgaben zuwies, zumal da wohl vielfach dieselben Räte, die als Mitglieder des Konsistoriums als geistliche Richter zu fungieren hatten, in der Kanzlei einzeln zu den kirchlichen Fragen herangezogen wurden, die dort vom Landesherrn erledigt wurden. Es ist auch sicher, daß gelegentlich die Tätigkeit der Konsistorien weiter gegangen war, als ihre Instruktion von Haus aus bestimmte. Nicht nur, daß wohl durch seine richterlichen oder schiedsrichterlichen Entscheidungen (z. B. über die Ansprüche der Pfarrwitwen) sich Grundsätze für die Praxis bildeten: wir wissen auch von einer Verfügung des Wittenberger Konsistoriums aus dem Jahre 1540, wonach der Pfarrer und der Kaplan von Schmiedeberg eine Zulage aus dem Gemeinen Kasten erhalten sollten. $\left.{ }^{1}\right)$

Aber jetzt kommen schon in den grundlegenden Konsistorialordnungen Erweiterungen vor. So bekommt das Mansfelder Konsistorium 1560 die Prüfung und Ordination der Geistlichen ${ }^{2}$ ), das neue Jenaer Konsistorium des ernestinischen Sachsens in den Verordnungen von 1561, 1568 und 1574 Anteil an der Bücherzensur, allgemeine Aufsicht über die Erhaltung der Kirchengüter in den einzelnen Gemeinden, und außerdem haben ihm die Superintendenten über ihre Visitationen $\mathrm{zu}$ berichten und seine Hilfe für die Besserung aller Mängel in Anspruch zu nehmen. ${ }^{3}$ ) In derselben Richtung steht auch die Brandenburgische Visitations- und Konsistorialordnung von 1573.4) Hier ist der Generalsuperintendent der Erbe der selbständigen bischöflichen Gewalt in allen eigentlich geistlichen Angelegenheiten: er prüit, ordiniert und instituiert die Geistlichen. Aber das Konsistorium ist

1) Sehling I, 1, 5̃ $\mathrm{M}$.

2) Ebd. I, 2, 195-197.

3) 1551 : ebd. I, 1, 230-233. - 1569: ebd. S. 233 ff. (Richter 2, 324 ff.). - 1574: ebd. S. 248 ff. (Richter 2, 395 ff.).

t) Richter 2, 358 ff. 
nicht wie unter Fürst Georg in Merseburg seine Behörde, sondern eine Behörde des Landesherrn. Und ein Kommissar des Konsistoriums wohnt den Prüfungen und Institutionen des Generalsuperintendenten bei. Generalsuperintendent und Konsistorium zusammen ordnen die Investitur an. Die neuen Pfarrer aber verpflichten sich durch einen schriftlichen Revers für ihre Amtsführung dem Konsistorium. Ihm steht auch die Kontrolle einzelner Teile der Vermögensverwaltung der Gemeinden $\mathrm{zu}$, und alle Inspektoren (Superintendenten) haben ihm über die Ergebnisse ihrer Visitationen zu berichten.

Aber bei alledem bleiben die Konsistorien doch in erster Linie Kirchengerichte. Das hat O. Mejer für das mecklenburgische schon längst festgestelit. ${ }^{1}$ ) Und das Konsistorium der Stadt Goslar ist lediglich E hegericht (1555). ${ }^{2}$ ) Aber auch für Brandenburg sieht Ad. Stölzel ${ }^{3}$ ) im Konsistorium einfach die kirchliche Parallele zum Kammergericht. Damit sei, sagt er, auch die geistliche Gerichtsbarkeit der landesherrlichen Gerichtsbarkeit eingereiht und mit ihr einer gemeinsamen Oberbehörde, den die Justiz verwaltenden Räten des Hofs, unterstellt worden. Daraus erkläre sich, daß das geistliche Ministerium später einen Teil des Justizministeriums gebildet habe. ${ }^{4}$ Wenn H. v. Mühler in seinem bekannten Buche ${ }^{5}$ ) eine ganz andere Darstellung gibt und im Konsistorium das Organ des gesamten Kirchenregiments sieht, so kommt das nur daher, daß er alles, was die Visitationsordnung von der landesherrlichen Visitationskommission sagt, ohne ein Wort darüber zu verlieren, auf das Konsistorium überträgt. So kann natürlich nur ein Zerrbild der Wirklichkeit herauskommen.

1) O. Mejer, Kirchen- und Konsistorialkompetenz nach mecklenburgischem Recht 1854, S. 77.

2) Richter 2, 163 if.

3) Ad. Stölzel, Brandenburg-Preußens Rechtsverfassung und Rechtsverwaltung 1, $224 \mathrm{f}$.

4) Eine Bemerkung, zu der freilich noch S. $334 \mathrm{f}$. hinzuzunehmen ist.

i) H. v. Mühler, Geschichte der evangelischen Kirchenverfassung in der Mark Brandenburg 1846, S. $60 \mathrm{ff}$., $80 \mathrm{ff}$. 
Die Anfänge der Konsistorialverfassung im luther. Deutschland. 13

Also auch da, wo die Konsistorien im Lauf der Zeit an der Aufsicht stärker beteiligt wurden, sind sie noch lange nicht Organe des gesamten landesherrlichen Kirchenregiments. In allen nord- und mitteldeutschen Gebieten gehen ja auch die alten außerordentlichen Visitationen mit ihrer ganzen prüfenden, organisierenden und anordnenden Arbeit fort. Die Kommissionen dafür aber ernennt der Landesherr unmittelbar. Sie sind seine, nicht des Konsistoriums Organe: nur ein oder der andere Konsistoriale, ein Kommissar oder auch nur ein Notar, wird neben ganz anderen Personen mit hereinberufen. Und außerdem gehen, wie es $z$. B. für Mecklenburg ausdrücklich bezeugt ist, in gewöhnlichen Zeiten die laufenden Sachen unmittelbar an den Hof. ${ }^{1}$ )

Dabei wird aber freilich zu bedenken sein, daß auf diesem ganzen geographischen Gebiet der Umfang solcher Geschäfte nicht allzu groß gewesen, ja auch die von den Kirchenordnungen geforderte Aufsicht der Konsistorien nicht sehr tief gegangen sein kann. Denn die landesherrliche Gewalt war viel $z u$ wenig entwickelt, die Macht der Patrone, vor allem des Adels, viel zu groß. Oberall standen sie der Aufsicht und der Zentralisation des Kirchenwesens im Wege. Sie konnten sich fast alles erlauben, was ehemals die Kircheneigentümer der alten Zeit geübt hatten, und zum Teil noch erheblich überbieten, was trotz aller Anstrengungen der Kirche der adlige Patron am Ende des Mittelalters gewagt hatte. Der Kampf, den die evangelischen Landesherrn dagegen sofort unternommen haben, hat im 16. Jahrhundert offenbar noch recht wenig Erfolg gehabt. ${ }^{2}$ )

II.

Diesem nord- und mitteldeutschen System gegenüber stellt nun die Württembergische Kirchenordnung Herzog Christophs einen ganz neuen

1) O. Mejer, Kirchen- und Konsistorialkompetenz nach mecklenburgischem Recht S. 79 f.

2) Darüber gedenke ich mich an anderem Ort auszusprechen. 
Typus dar. Auch das wird zurzeit verkannt. Man sieht in ihr ausdrücklich eine Nachbildung der kursächsischen Konsistorialverfassung und billigt ihr nur die Bedeutung $z u$, daß sie zuerst eine kirchliche Zentralbehörde für das ganze Land geschaffen und damit auf Kursachsen zurückgewirkt habe. ${ }^{1}$ )

Aber auch das ist nicht richtig. Einheitliche Konsistorien haben andere Länder schon vor Württemberg oder bald darauf und ganz unabhängig von ihm gehabt. So war es von den erobernden Fürsten schon Braunschweig-Wolfenbüttel 1543 zugedacht gewesen; so hatten es die Theologen der Lätarekonferenz von 1544 für das albertinische Sachsen vorgeschlagen. So war es wirklich in Mecklenburg 1552, in der Grafschaft Mansfeld schon vor 1560, im ernestinischen Sachsen 1561, so vor allem in Kurbrandenburg. Und alle diese Ordnungen zeigen nicht den mindesten Einfluß der württembergischen Kirchenordnung.

Also diese Einheitlichkeit ist nichts besonderes. Württemberg war für mehrere Konsistorien einfach schon zu klein. Seine Kirchenbehörde heißt aber auch nicht Konsistorium oder Kirchengericht, sondern Kirchen $r a t$, und vor allem: sie hat ganz anderes Gepräge. Während die nord- und mitteldeutschen Konsistorien aus dem Bedürfnis nach Kirchengerichten herausgewachsen waren und die Analogie zu den fürstlichen Kammer- oder Landgerichten bildeten, ist der württembergische Kirchenrat aus den Visitationskommissionen der früheren Jahrzehnte entstanden und stellt das Analogon zum herzoglichen Rat als der Behörde der neuen weltlichen Zentralverwaltung dar.

Schon in dieser Analogie liegt ein interessanter Vorgang. Von den burgundischen Niederlanden aus war

1) Richter, Kirchenverfassung S. 121, Rieker S. 175, 177. U. Stutz in v. Holtzendorff-Kohlers Enzyklopädie der Rechtswissenschaft ${ }^{B} 2,891$ hebt wenigstens ganz richtig hervor, daß Württemberg im Unterschied von der sächsischen Kirchenordnung von 1580 keine Unterkonsistorien gehabt habe. 
bekanntlich diese Zentralverwaltung durch Maximilian I. für Osterreich übernommen worden, und von da aus drang sie nun auch im übrigen Deutschland vor. Auch Herzog Ulrich, der Vater Herzog Christophs, hatte sie nach seiner Wiedereroberung des Landes 1534 in Württemberg eingeführt, soweit es die kleinen Verhältnisse des Landes verlangten und erlaubten. ${ }^{1}$ ) Während bisher die Räte des Herzogs nach dem alten System meist im Lande zerstreut auf ihren Adelssitzen oder den lehensherrlichen Burgen gewohnt hatten und nur für bestimmte Angelegenheiten einzeln an das Hoflager berufen worden waren, hatte Ulrich jetzt eine größere Anzahl juristischer und adliger Räte in einer ständigen Behörde mit kollegialer Verfassung, dem nachmals sogenannten Oberrat, vereinigt und zugleich die ganze Finanzverwaltung einer ebenso organisierten Behörde, der Rentkammer, übertragen. Für die kirchliche Verfassung hatte er erst kurz vor und noch während des Schmalkaldischen Krieges die ersten Versuche gemacht. Er hatte nach schweizerischem Vorbild die alten Ruralkapitel mit ihren selbstgewählten Dekanen an der Spitze erhalten wollen und nur ihre Bezirke im Anschluß an die Ämter des Landes umgelegt. Wie in der evangelischen Schweiz sollten sie auch hier auf zwei jährlichen Synoden die Aufsicht und Zensur über ihre Mitglieder ausüben und Einhelligkeit im Predigtamt und in den Kirchengebräuchen erhalten. Landesherrliche Superattendenten wurden als Aufsichtsinstanz über diesen Kapiteln in Aussicht genommen. ${ }^{2}$ ) Daneben waren die Visitationskommissionen sächsischer Art geblieben, neben der weltlichen Zentralverwaltung ein archaistischer Rest der alten Verwaltungsformen, unständig, in Zwischenräumen vom Herzog zusammen-

1) Vgl. Fr. Wintterlin, Geschichte der Behördenorganisation in Württemberg $1,24 \mathrm{ff}$.

2) Vgl. Herzog Ulrichs Synodalordnung von 1547 bei Sattler, Geschichte des Herzogtums Württemberg unter der Regierung der Herzoge Bd. 3, Anh. S. 277; Reyscher, Vollständige ... Sammlung der württembergischen Gesetze Bd. 8 (herausgegeben von Th. Eisenlohr) S. 80 ff. Richter 2, 92. 
berufen, aus Theologen, Adligen und Vertretern der städtischen Ehrbarkeiten bestehend.

Da bildete Herzog Christoph auch dieses kirchliche Regimentsorgan nach den neuen Grundsätzen um. ${ }^{1}$ ) 1553 wurde ein ständiges Kollegium herzoglicher Beamten, der Visitationsräte, unter einem eigenen Direktor eingerichtet, und in der großen Kirchenordnung von $155^{2}$ ) erhält diese Behörde den Namen Kirchenrat, die kirchliche Parallele zum weltlichen Oberrat, mit ihm zusammen unter der einheitlichen Spitze des Landhofmeisters. Die Theologen haben alle Personalangelegenheiten der Geistlichen und Schullehrer, Prüfung, Anstellung, Aufsicht und Disziplinargewalt bis zur Absetzung. Die politischen Räte haben überall für die Wahrung der herzoglichen Patronats- und Vogteirechte einzutreten, das soeben einheitlich organisierte Kirchengut und seine Verwaltung im Land zu überwachen, und ein Teil von ihnen, die später sog. geistliche Kammer, die Parallele zur Rentkammer, hat die Uberschüsse einheitlich zu verwalten.

Zugleich ist die Verfassung nach unten ausgebaut. Die Kapitel sind verschwunden. Die norddeutschen Superintendenten sind aufgenommen, vom Herzog ernannt und in drei Stufen entwickelt: den Spezialsuperintendenten, die bald in allen Amtsstädten sitzen, den

1) Herzog Christophs Visitationsordnung von 1533 bei Sattler Bd. 4, Anh. S. 54 ff., Eisenlohr S. 100 ff. Die Ordnung beginnt mit "Und". Dieser Anfang findet sich aber nach gütiger Mitteilung des Archivdirektors Herrn Dr. v. Schneider schon in der Handschrift des Kgl. Staatsarchivs, die dem Druck Sattlers zugrunde liegt. Sie ist vom Herzog eigenhändig unterschrieben, hat also wohl ursprïnglich nach der Vermutung Dr. v. Schneiders mit den anderen kirchlichen Ordnungen des Jahres 1553 zusammen e in e Vorlage gebildet und ist nur nicht wie die sog. kleine Kirchenordnung sogleich gedruckt worden.

2) Ich benutze den verbreitetsten Druck bei Eisenlohr S. $106 \mathrm{ff}$. Auch Richter 2, 198 gibt die Stücke, die hier in Betracht kommen. - Die Entstehung des Kirchenrats aus den Visitationskommissionen Herzog Ulrichs hat schon Eisenlohr in der vortrefflichen Einleitung zum 9. Bande von Reyschers Sammlung verfolgt. Daraus Richter, Kirchenverfassung S. $121 \mathrm{f}$. und alle Neueren. 
vier Generalsuperintendenten und darüber dem Propst der Stuttgarter Stiftskirche. Aber diese Superintendenten haben doch ein anderes Gepräge als die sächsischen. Sie knüpfen nicht an den kirchlichen Einzelrichter des Mittelalters an, sind vielmehr ganz aus dem System der alten Visitationen herausgewachsen. Sie haben keinerlei richterliche Aufgaben, haben nichts mit Prüfung der neuen Geistlichen zu tun, sind vielmehr lediglich mit der Aufsicht und regelmäßigen Visitation ihrer Bezirke und der damit verbundenen seelsorgerlichen Kirchenzucht betraut, die Spezialsuperintendenten für die Amtsführung und das kirchliche wie sittliche Leben ihrer Geistlichen, Lehrer und Gemeinden, die Generalsuperintendenten für die Speziale. Und dieses System der Visitation ist dann wieder mit dem Kirchenrat verknüpft und ihm untergeordnet dadurch, daß die Spezialsuperintendenten ihre Visitationsberichte an die Generalsuperintendenten, diese das Ergebnis aller Berichte an den "Gemeinen Konvent der Superintendenz beim Kirchenrat " bringen, eine Verbindung von Kirchenrat, Generalsuperintendenz und Propst von Stuttgart unter dem Vorsitz des Landhofmeisters, den später sog. Synodus. Hier werden dann alle Schäden, die sich bei den Visitationen ergeben haben, besprochen, Maßregeln zur Besserung und Strafen beschlossen oder dem Herzog vorgeschlagen.

Dieses ganze System hat also mit der geistlichen Gerichtsbarkeit so gut wie nichts zu tun. Für die Ehesachen, die in Sachsen und seinem Bereich zunächst die Hauptmasse aller Geschäfte ausmachten, war in Württemberg ein besonderes Gericht bestellt worden. Die Ordination, die in Nord- und Mitteldeutschland zum Teil den Konsistorien übertragen wurde, bestand in Württemberg nicht. ${ }^{1}$ ) Auch die Prozesse über Pfründen fallen weg, weil der Kirchenrat den größten Teil der Stellen selbst vergibt und für die noch vorhandenen fremdherr-

1) Auch das ist ein Punkt, in dem sich die württembergischen Formen an das schweizerische Gebiet anschlossen. Ebenso liegt es $\mathrm{ja}$ auch in der Liturgie.

Historische Zeitschrift (102. Bd.) 3. Folge 6. Bd. 
lichen Patronate strenge die Bestätigung übt. Die Kirchenzucht aber hat rein kirchliches Gepräge: der Bann wird nach lutherischen Ideen geübt, ohne Geld- und Leibesstrafen. So ist der Kirchenrat eine reine Verwaltungsund Aufsichtsbehörde, und darum fallen in Württemberg künftig die unständigen Visitationskommissionen ganz weg, während sie im Norden neben den Konsistorien fortbestehen. Es ist darum ganz bezeichnend, daß die norddeutschen Behörden Konsistorien oder Kirchengerichte heißen, Württemberg dagegen seinen Kirchenrat hat. Wo man in nächster Zeit auch in anderen Ländern diesem Namen begegnet, da ist er fast ausnahmslos ${ }^{1}$ ) ein Zeichen dafür, daß dort die württembergische Organisation übernommen worden ist oder ihren Einfluß ausgeübt hat.

In der Tat ist der Kirchenrat der Markgrafschaft Baden-Durlach ${ }^{2}$ ) ebenso wie der, den Kurfürst Fried-

1) Eine Ausnahme bildet in den von Richter gesammelten Kirchenordnungen, soviel ich sehe, nur die Niedersächsische (Lauenburgische) Kirchenordnung von 1585 (Richter 2, 469 ff.), wo die Uberschrift (S. 471 a) lautet: "Von dem Kirchenradt oder Consistorio." Damals hatte sich der Titel Kirchenrat durch den Einfluß der württembergischen Kirchenordnung in Norddeutschland schon mehrfach verbreitet.

2) Das kann ich vorläufig nicht eigentlich beweisen. Ich habe über die Einrichtung des badischen Kirchenrats nur die eine Notiz bei K. Fr. Vierordt, Geschichte der evangelischen Kirche in dem Großherzogtum Baden 1, 429 gefunden, und da ist keine Quelle genannt. Anfragen in Karlsruhe bei dem Großherzoglichen General-Landesarchiv und bei einem Mitglied des Großherzoglichen Kirchenrats haben kein Ergebnis gebracht. Allein da Baden z. B. in der Liturgie ganz dem württembergischen Vorbild sich anschließt und da J. Andreä auch bei der badischen Kirchenorganisation beteiligt war, so wird anzunehmen sein, daß der badische Kirchenrat in der Tat eine Nachahmung des württembergischen sei. Das Großherzogliche General-Landesarchiv schreibt mir außerdem, indem es meine Vermutung ausdrücklich bestätigt: „Aus Akten Baden Generalia Fasz. 1287 geht hervor, $\mathrm{daB}$ sich die baden-durlachische Regierung 1575 vom Herzog von Württemberg zwecks einer neuen Behördenorganisation eine württembergische Ordnung zusenden ließ. Nachweislich wurde auch ein Teil derselben ausgiebig für die Ordnung von 1577 be- 
rich III. von der Pfalz bei seinem Ubergang zum sog. Kalvinismus 1564 eingerichtet hat ${ }^{1}$ ), nichts als eine Nachbildung des württembergischen. Und der braunschweigwolf enbüttelische Kirchenrat von $1569^{2}$ ) zeigt fast nur noch darin die Einwirkung des norddeutschen Typus, daß er "Kirchenrat oder Konsistorium" heißt. Auch die Einrichtung der Superintendenz schließt sich wörtlich an die württembergische Kirchenordnung an. Schwächer war der Einfluß auf Lippe $1571^{3}$ ), viel stärker wieder auf $\mathrm{H}$ e n n e be r $\mathrm{g}^{4}$ ), dessen Fürst der Schwager Christophs war.

Am interessantesten aber ist der Einfluß des württembergischen Systems auf Kursachsen und seine Kirchenordnung von 1580. Wie in Baden, Wolfenbüttel, Lippe und Henneberg, so hat auch in Sachsen der Tübinger Kanzler Jakob Andreä das Mittelglied gebildet. Vier Jahre lang, 1576-1580, hat er in sächsischen Diensten gestanden und die Kirchenordnung neu gestalten dürfen.

Der wïrttembergische Einfluß auf Sachsen tritt in einzelnen bedeutsamen Verordnungen schon seit $1578^{5}$ ), vor allem aber in der großen Kirchenordnung von $1580^{6}$ ) hervor. Ganze Abschnitte sind hier völlig oder zum Teil wörtlich aus der Kirchenordnung Herzog Christophs

nutzt. Eine Kirchenratsordnung allerdings ist nicht vorhanden, und es läßt sich auch nicht erweisen, daß die württembergische mit übersandte einer etwaigen badischen als Vorbild diente, da in dem württembergischen Exemplar keinerlei Korrekturen angebracht sind, wie dies bei den übrigen eingesandten württembergischen Ordnungen, die als Muster für badische Ordnungen übernommen wurden, der Fall ist." lch bemerke dazu, daß nach Vierordt der Kirchenrat irüher eingerichtet zu sein scheint.

1) Richter 2, $276 \mathrm{ff}$.

2) Ebd. S. 322 ff.

3) Ebd. S. 338 b f.

4) Sehling I, 2, 285 ff. und die Einleitung S. $273 \mathrm{ff}$.

5) Vgl. die Mitteilungen Sehlings I, 1, $113 \mathrm{f}$.

6) Sehling I, 1, 359 ff. Auch Richter 2, $401 \mathrm{ff}$., doch unvollständig. Vgl. auch F. Ludwig, Zur Entstehungsgeschichte der Lokalvisitationen, des "Synodus" und des Oberkonsistoriums in Sachsen: Kirchenordnung von 1580 (Beiträge zur sächsischen Kirchengeschichte 21, 1 ff. 1908). 
entlehnt. Ihr Einfluß äußert sich vor allem in folgenden Punkten:

1. Die Synoden, die bisher die Superintendenten an ihrem Sitz hätten halten sollen, mindestens in letzter Zeit aber großenteils nicht mehr gehalten hatten, werden durch regelmäßige Visitationen am Ort der Gemeinden und Geistlichen ersetzt.

2. Ober den Superintendenten wird das Amt der Generalsuperintendenten eingerichtet, die die Superintendenten beraten und visitieren und zugleich für sie eine Zwischeninstanz zum Konsistorium bilden sollen.

3. Die Konsistorien erhalten die Prüfung (Ordination) und Investitur aller patronatisch nominierten Geistlichen, in den einzelnen Fällen größeren Einfluß auf die Besetzung der geistlichen Stellen, die Kontrolle der Patrone und schärfere Aufsicht über die Amtsführung der Geistlichen.

4. U ber den Konsistorien wird ein Oberkonsistorium errichtet, das jedoch zugleich das bisherige Meißener Konsistorium in sich aufnimmt und so zugleich das Konsistorium eines bestimmten Kreises bleibt.

5. Bei dem Oberkonsistorium wird der Generalsynodus eingerichtet, die vollkommene Nachbildung des württembergischen Konvents der Superintendenz beim Kirchenrat, der seinerseits wiederum von Sachsen später den Namen Synodus übernommen hat.

6. Das bisherige System der außerordentlichen Visitationen durch landesherrliche Kommissionen wird damit ersetzt durch den württembergischen Apparat regelmäßigen Visitierens durch ständige Beamte.

Dieselben Ordnungen hat dann der Kurfürst auch in den ernestinischen Gebieten eingeführt, die er damals vormundschaftlich regierte. ${ }^{1}$ )

Durch den württembergischen Einfluß ist also das sächsische Kirchenwesen stärker zentralisiert, der Einfluß des Landesherrn erheblich erweitert, die Macht des Adels eingeschränkt und das Superintendentenamt einiger-

1) Sehling I, 1, 76 f. 81 ff. 
maßen entwertet worden, obwohl ihm in den Visitationen ein neues bedeutsames Moment zugeführt worden ist. Denn der Schwerpunkt liegt jetzt doch in den zentralen Behörden, die mit allen Aufgaben des Kirchenregiments betraut, zugleich aber immer noch Kirchengerichte im früheren Sinn geblieben sind. Doch erstreckte sich die Gewalt des Konsistoriums oder des Oberkonsistoriums nur auf das alte sächsische Gebiet sowie die ehemaligen ernestinischen Landesteile, nicht auch auf die Lausitz und die früheren bischöflichen Territorien von Merseburg und Naumburg-Zeitz. Sie unterstanden auch kirchlich im wesentlichen einfach der kurfürstlichen Kanzlei. ${ }^{1}$ )

Allein auch im altsächsischen Gebiet war die württembergische Zentralisation vorerst gar nicht durchzuführen. In Württemberg gab es keinen landsässigen Adel und keine halbautonomen Städte. Von den geistlichen Stellen standen hier etwa $4 / 5$ bis ${ }^{5} i_{6}$ unter dem Patronat des Herzogs, der Rest fast nur unter ausländischen, meist altgläubigen Patronen, deren Kandidaten der Kirchenrat scharf prüfte, zuließ und verpflichtete. Hier war es darum auch möglich gewesen, aus den sämtlichen Stellen herzoglichen Patronats und den eingezogenen geistlichen Instituten ein allgemeines Kirchengut unter fürstlicher Verwaltung zu schaffen, für das ein ganzer Apparat von Beamten bestand.

In Sachsen dagegen lagen die Verhältnisse ganz anders. Das Land war für diese Zentralisation bei den damaligen Verhältnissen schon viel $z u$ groß. Die Arbeitslast, die damit den Oberbehörden erwuchs, war kaum zu bewältigen. Vor allem aber kamen politische Faktoren dazu. Alle bisherigen Versuche, die Visitationen an Ort und Stelle einzuführen, das Kirchengut der Pfarreien stärker unter die Kontrolle der Konsistorien zu stellen u. a., waren von vornherein durchaus nicht nur an den Kosten, sondern zumal an der Rücksicht auf den Adel gescheitert, der, wie in ganz Norddeutschland, die Kirchen-

1) G. Müller in den Beiträgen zur sächsischen Kirchengeschichte 9, 136 f. 144. 
güter an sich zog, den Kirchenkassen kurzer Hand „Darlehen" entnahm und der Visitation sich nicht zu unterziehen gedachte. Auch die politische Zentralregierung, die sächsische Geheimeratskanzlei, fühlte sich durch das neue Oberkonsistorium in ihrem Einfluß auf die kirchlichen Verhältnisse beeinträchtigt, was doch wohl auch ein Beweis für den Umfang ist, in dem bisher die kirchlichen Angelegenheiten trotz aller Konsistorien unmittelbar an den Landesherrn und seine Kanzlei gegangen waren. So wollte denn das dem Adel so unwillkommene System der Visitationen nicht gedeihen und erlahmte bald völlig. Der Generalsynodus schlief zeitenweise ein, und das Oberkonsistorium wurde schon 1588 aufgelöst, um erst 1606 wieder zu erstehen.

Den Ländern, in denen der württembergische Kirchenrat nachgeahmt worden ist, schließt sich am Anfang des 17. Jahrhunderts Hessen an, wo Landgraf Moritz 1610 der bisherigen Synodalverfassung ein Ende machte und "den abgesonderten Kirchenrat und Konsistorium " schuf. ${ }^{1}$ ) Die Vereinigung beider Titel im Namen der neuen Behörde ist bezeichnend. Denn sie verband die regimentlichen Befugnisse des württembergischen Kirchenrats mit den richterlichen der norddeutschen Konsistorien.

Aber auch Brandenburg bekam wenigstens für kurze Zeit eine ähnliche Schöpfung.

Im Zusammenhang mit dem Obertritt Johann Siegmunds zum reformierten Bekenntnis wurde 1614 der Kirchenrat eingerichtet, der die landesherrlichen Patronate zu besetzen, die Inspektoren zu ernennen, kirchliche Anordnungen z. B. in liturgischen Dingen zu treffen und die Visitationsberichte der Inspektoren $z u$ bearbeiten hatte. Beide Mitglieder der Behörde, Präsident wie Assessor, waren reformiert. ${ }^{2}$ ) Es war ein Schlag gegen das lutherische Konsistorium, und der Gedanke liegt nahe, daß der pfälzische Kirchenrat das Vorbild gewesen sei. Das Konsistorium schrumpfte wieder fast $\mathrm{zu}$ einem

1) Heppe, Kirchengeschichte beider Hessen 2, 40.

2) H. v. Mühler S. 129. 
Die Anfänge der Konsistorialverfassung im luther. Deutschland. 23

Ehegericht zusammen. Freilich, der Kirchenrat wurde schon 1618 wieder aufgehoben. Aber seine Funktionen fielen nun nicht wieder an das Konsistorium zurück, sondern wurden teils dem Geheimen Rat übertragen, teils vom Landesherrn unmittelbar oder mit Hilfe des Geheimen Rats oder besonders bestellter Kommissare erledigt.

So sind also die Konsistorien erst ganz allmählich im 16. Jahrhundert und auch gar nicht überall das geworden, was wir in ihnen von Anfang an zu sehen gewohnt sind, Organe des landesherrlichen Kirchen regiments. Schon die Bedürinisse der Aufsicht vor allem haben z. T. auf diesen Weg geführt. Vor allem aber hat in einer Reihe hervorragender Territorien das württembergische Vorbild den Anstoß dazu gegeben.

Mit dem Beginn des Dreißigjährigen Krieges endigt diese Anfangsperiode der lutherischen Kirchenverfassung. Im Krieg zerfällt sie fast überall. Nach dem Frieden aber kommen neue Verhältnisse. Die Aufklärung bereitet sich vor, setzt neue Ziele und wandelt auch die Formen des Kirchenregiments um. Sie zu verfolgen ist nicht mehr meine Aufgabe.

\section{Anhang.}

\section{Der Senatus ecclesiasticus in den Vorschlägen der Leipziger Lätarekonferenz 1544.}

(Bei Sehling, Kirchengesetzgebung unter Moritz von Sachsen, S. 121 ff.)

Unter den Gutachten, die der Ausgestaltung des albertinisch-sächsischen Kirchenwesens vorangingen, nimmt das der angesehensten Theologen des Landes, die am 23. März in Leipzig zusammentraten, besonderes Interesse in Anspruch. Sehling, der es zum erstenmal bekannt gemacht hat, hat es zugleich eingehend besprochen (S. 1 ff.) und dabei namentlich der Institution des Senatus ecclesiasticus oder, wie er im Anschluß an ein anderes Gutachten ibersetzt, des Kirchenrats 
seine Aufmerksamkeit zugewandt. $\operatorname{Er}$ hebt (S. 3) folgende Punkte hervor: 1. In ihm seien die Seniores oder Kirchväter mit dem Pfarrer vereinigt. 2. Er übernehme die $Z u c h t$ in der Einzelgemeinde. 3. Er richte zugleich in Verbindung mit dem Superintendenten die geringeren Delikte der Pfarrer, bilde also neben dem Superintendenten eine Art Aufsichtsinstanz über die Pfarrer. 4. Zugleich habe er eine Kontrolle über die Verwaltung des Kirchenvermögens: die Kastenherren hätten ihre Rechnung künftig vor ihm zu legen. 5. Er sei aber endlich auch an der Ernennung der Pfarrer beteiligt.

Wenn ich ihn recht verstehe, sieht Sehling in diesem Kirchenrat eine Einrichtung jeder Einzelgemeine in Stadt und Land. ${ }^{1)}$ Darin folgt ihm E. Brandenburg. ${ }^{2}$ ) Seine Beschreibung des Amtes ist überhaupt dieselbe wie bei Sehling, nur $\mathrm{da} B$ er nun aus dem ganzen Gutachten viel schärfer die Eigenart der Verfassungsideale der Theologen herauszuarbeiten sucht: im Gegensatz zum Episkopalsystem des Fürsten Georg und dem Territorialprinzip der herzoglichen Räte vertrete es das Gemeindeprinzip, das Selbstregiment der Gemeinden und ihrer Vertretung in einem gemeinsamen Kollegium, dem Konsistorium.

Ich fasse die Sache anders auf und führe zunächst das Material vor.

Das Gutachten der Konferenz (I) $\left.{ }^{3}\right)$ will der wachsenden Bosheit neue Mittel entgegenstellen lassen, die zugleich geeignet wären, dem Herzog wie dem Konsistorium viel Arbeit abzunehmen, Kosten zu ersparen und die Verhängung des rechten Banns zu erleichtern. Der Weg dazu wäre, [1] daß den Superintendenten in jeder Stadt ihrer Superattendenz und ihrer Pfarreien etwa zwölf Personen als Älteste beigeordnet würden: der Prediger, verständige Ministri, etliche gottesfürchtige Männer vom Rat und aus den Vornehmsten der Gemeine. Diese sollten neben dem Superintendenten Macht haben, alle Gebrechen und Irrungen in der Kirche in Lehre und Leben zu erforschen, die Schuldigen vor sich zu bescheiden,

1) Vgl. namentlich auch S. 4-6, wo er von Gemeindeältesten und dem Gemeindeprinzip spricht und in diesen Zusammenhang das Gutachten und seinen Kirchenrat stellt.

$\left.{ }^{2}\right)$ E. Brandenburg, Die Entstehung des landesherrlichen Kirchenregiments im albertinischen Sachsen (Hist. Vierteljahrschr. 4, 195 ff., 1901).

3) Ich benutze die Ziffern, die Brandenburg S. 196 Anm. 2 eingeführt hat. 
$z u$ vernehmen und dazu zu bringen, daß sie für ihre öffentlichen Laster öffentlich Buße täten, für die geheim gebliebenen private vor den Ältesten allein. Hartnäckige, unbußfertige Sïnder wären dem Konsistorium zu weiterem Verfahren anzuzeigen, das dann nicht mehr mit bloßer seelsorgerlicher, sondern mit wirklicher Strafgewalt ausgestattet wäre.

Das Gutachten kommt dann [2] auf diesen Vorschlag zurück in dem Abschnitt "Ordnung des Consistorii" S. 143. Die Verfasser meinen $z$ war, ein einziges Konsistorium werde für das Land nicht genügen, zumal wenn von den Pfarrern und ihren zugeordneten Ältesten in den Städten (von denen oben Meldung getan sei) nicht aller Ernst angewendet werde. Doch möge man vorerst einmal einen Versuch mit e inem Konsistorium machen in Hinsicht auf den Senatus ecclesiasticus, der neben den Superintendenten dem Konsistorium viel Mühe ersparen werde.

Endlich erscheint [3] der Kirchensenat auch noch in dem Abschnitt, der sich mit der Visitation und Inquisition befaßt, S. 149: Ehe ein Diffamat dem Konsistorium überwiesen werde, sollen sich die Visitatoren erkundigen, ob er zuvor durch den Pfarrer oder einen andern unter vier Augen ermahnt, dann nach Verachtung solcher Ermahnung vor zwei Ältesten oder Kirchvätern vorgenommen und nach weiterer Verachtung dem Senatui ecclesiastico des Orts angezeigt und von ihm öffentlich mit Gottes Wort gestraft und ermahnt worden sei.

In einem etwas späteren Gutachten, das etwa vom Ende April 1544 stammt (III), kommen die Theologen auf ihren Gedanken zurück.1) Solche Senatus ecclesiastici sollen in allen Städten bestehen aus Kirchendienern, Ratsmännern und einer Anzahl der angesehensten Bürger, zusammen acht bis zehn Personen, die als Zensoren und Zuchtmeister zu walten hätten.

Ein drittes Gutachten dreier Superintendenten (II), das $z w i s c h e n$ den beiden ersten liegt ${ }^{2}$ ), findet $z$ wei Möglichkeiten für die Handhabung des Banns: entweder überläßt man ihn dem Konsistorium, oder könnte man ihn durch jeden Superintendenten samt dem Senatu[i] ecclesiastico des Orts, da der Superintendent Pfarrherr sei, über alle Unbußfertigen des Bezirks seiner Superattendenz auf Antrag des Pfarrherrn und der Kirchväter verhängen lassen. Man gewänne also ein Kirchengericht, das in Händen des Konsistoriums oder der

1) Sehling S. $20 \mathrm{f}$.

s) Brandenburg S 222 (u. 1), S. 223: "Nota." 
Superattendenten samt ihren Senatibus läge. Beide Behörden, das Konsistorium wie der Superattendent mit dem kirchlichen Senate seines Bezirks, repräsentierten dann die allgemeine Kirche und richteten so an Statt und im Namen aller andern Kirchen.

Weiter handelt auch das Gutachten einiger Superintendenten vom Juni 1545 (IX) noch einmal von dem Senatu ecclesiastico oder Kirchenrat. ${ }^{1}$ ) Wiederum wird empfohlen, ihm den Bann zu übertragen. Sonst käme dieser Bann nicht auf: denn die Pfarrer wollten das Odium und die Kosten des Verfahrens nicht allein tragen; ein solcher Senat dagegen könnte ihnen die Fortsetzung abnehmen und das Verfahren zum Ziele führen. ${ }^{2}$ ) Damit wäre auch der Vorteil verbunden, daß jeder Pfarrer seine Aufseher bei sich hätte und nichts aus eigenem Gutdünken anfangen, anderseits aber auch für Nachlässigkeit und Faulheit gestraft werden könnte.

Diese Stellen sind durchaus einhellig. Sie bezeugen, daß es sich nicht um einen Gemeindekirchenrat handelt, den jeder Pfarrer auch auf dem Lande neben sich hätte ${ }^{3}$ ), sondern um ein Bezirksgericht für geistliche Vergehen, das nur in den Städten ${ }^{4}$ ), insbesondere den Sitzen der Superintendenten, bestehen soll. ${ }^{5}$ ) Auch der letzte Satz, den ich aus IX angeführt

1) Sehling S. $52 \mathrm{f}$.

2) Vgl. S. 52 ü. d. M.: ,dieweil die pfarrherrn nicht allein mit haB und neit, auch mit anschuldigung und anweisung derer, so zu verbannen, und allerlei unkosten ... beschwert ... sein wollen, da sonst durch einen senatum ecclesiasticum auf der kirchen unkosten solches unnachleßlich seine fortsetzung haben und also demnach jedermann zu guter besserung dienlich sein wurde."

2) Nur in der Anmerkung füge ich noch bei, was Sehling S. 65 aus den Ergebnissen der Verhandlungen zwischen Fürst Georg und den Theologen mitteilt: Der Senatus ecclesiasticus wird abgelehnt. Aber den Superintendenten und Pfarrherrn sollen die Räte der Städte, wenn die Ermahnungen der Superintendenten allein nichts fruchten, etliche zuordnen, damit diese zusammen mit dem Superintendenten oder Pfarrherrn nochmals ermahnen.

4) Auch in I, 3, S. 149 ist der Senatus ecclesiasticus des Ortes eben der Senat, zu dessen Bezirk der Ort gehört, wenn nicht vielmehr Ort einfach $=$ Stadt ist. Vgl. z. B. S. 140 M.: „Ein $y$ der superattendent soll seins orts vleißig uffsehen haben " usw.

$\left.{ }^{5}\right)$ Hier finde ich die einzige Unklarheit. Nach I, 1 u. III müßte man meinen, daß die Senate in jeder Stadt bestehen 
habe, bedeutet nicht, daß der Kirchenrat "neben dem Superintendenten eine Art Aufsichtsinstanz über die Pfarrer bilde" (Sehling S. 3); er bezieht sich vielmehr lediglich auf das Bannverfahren: dem Pfarrer soll das Odium des alleinigen Vorgehens abgenommen, es soll ihm aber auch erschwert werden, willkürlich vorzugehen oder die Laster ungestraft zu lassen. In erster Linie wird sich auch das auf die städtischen Pfarrer, also die Superintendenten beziehen. Aber die vom Land haben auch Anteil daran, insofern als der Senat ja auch bei ihnen Aufsicht uibt und ihre Anzeigen entgegennimmt.

Mitglieder des Senats sind der Superintendent als Pfarrer des Orts, die übrigen Geistlichen der Stadt und eine Anzahl Personen aus dem Rat und der städtischen Aristokratie. Er hat den sittlichen Vergehen, die den Bann zur Folge haben könnten, nachzuspüren $(l, 1)$; ihm werden aber auch von den Pfarrern des Bezirks, also auch der Landgemeinden, die unbußfertigen Sünder gemeldet und zu weiterem Verfahren übergeben (I, 3, II, IX). Haben auch seine Ermahnungen keinen Erfolg, so werden die Sünder dem Konsistorium zum Ausschluß aus der Kirche übergeben $(I, 1,3)$, sofern man nicht dem Kirchensenat selbst den Bann zu übertragen gedenkt (II, IX).

Dabei ist ein ganz regelmäßiger Gang vorgesehen: $1 . \mathrm{Ge}$ heime Ermahnung durch den Pfarrer, 2. ebenso durch den Pfarrer und zwei Âlteste oder Kirchväter des Orts, 3. öffentliche Strafe seelsorgerlicher Art vor dem kirchlichen Bezirksgericht, 4. öffentlicher Bann durch das Konsistorium. Da von "Ältesten" in den Landgemeinden sonst gar nicht die Rede ist, so wird bei 2. nur an städtische Verhältnisse zu denken sein. Die Ältesten sind dann eben einzelne Personen, die zum Kirchensenat gehören.

Außer der Sittenzucht haben diese Gerichte nach I und IX auch Anteil an der Kontrolle der Verwaltung der Gemeinen Kasten. ${ }^{1}$ ) Auf Grund einer früheren, aber vielfach nicht beobachteten „sächsischen Ordnung“2) sollen

sollten, der Superintendenturbezirk aber unter Umständen mehrere Städte umfassen könnte. Nach II aber sieht es aus, als ob er nur in de n Städten, die Sitze von Superintendenten sind, bestehen sollte.

1) Sehling S. 140 u. 52.

2) Ich weiß nicht, was damit gemeint ist. Die „Landesordnung "Herzog Moritzens (Sehling, Kirchenordnungen I, 1, 286 ff.) 
die Superintendenten diese Aufsicht üben; die Verfasser leiten daraus aber weiter ab, daß die Kastenherrn ihre Rechnung vor der Obrigkeit, Rat, Pfarrer und Senatus ecclesiasticus tun sollen. Auch IX wünscht einen solchen Anteil des Senats (S. 52 u.), damit die Gelder des Kastens ihrer wirklichen Bestimmung gemä $\beta$ verwendet werden. Bei alledem aber handelt es sich wieder nur um die Städte: die Gefahr ist nach IX, daß durch die unrechtmäßigen Ausgaben "die Armen der Stadt“ geschädigt würden ${ }^{1}$ ), weshalb auch vom Pfarrer und von den Kirchendienern des Orts die Rede ist, d. h. den übrigen Geistlichen, wie sie im allgemeinen wohl nur in den Städten neben dem Pfarrer vorhanden sind. Auch die Gemeinen Kasten sind ja, wenn ich recht sehe, im wesentlichen tatsächlich nur eine Einrichtung der Städte.

Dagegen ist es ein Irrtum, wenn Sehling und Brandenburg dem Senat einen Anteil an der Pfarrwahl zusprechen. Sie denken dabei an die Stelle im Lätaregutachten (S. 123 1. d. M.), nach der die Berufung der städtischen Pastoren beim Rat, die der übrigen bei den Patronen "samt den Vornehmsten der Gemeine" stehen soll. Daß aber diese "Vornehmsten" nicht "die Gemeindeältesten" (Sehling S. 202 zu Anm. 3) oder der Kirchensenat sind, wird, von allem anderen abgesehen, schon daraus klar, daß nach $\mathrm{l}, 1$ die Vornehmsten der Gemeine nur mit dem Prediger und einigen Ratsherren zusammen den Senat der $\ddot{A} l t e s t e n$ bilden. Es handelt sich also lediglich um besonders angesehene Männer der Landgemeinden, keinen Gemeindekirchenrat.

So bleibt also als die ganze Aufgabe des Senats, daß er mit dem Superintendenten zusammen das Zuchtgericht für seinen Bezirk und die Kontrolleinstanz für den Gemeinen Kasten der Stadt bilde. Ich glaube darum auch nicht, daß das Lätaregutachten so, wie Brandenburg es meint, das Gemeindeprinzip, die Unabhängigkeit der Kirche von jeder staatlichen Fessel vertrete. Doch gehe ich darauf hier nicht näher ein, sondern bemerke nur kurz folgendes:

1. Dorfälteste kommen in dem Gutachten, wenn ich recht sehe, iuberhaupt nicht vor; nur bei der Visitation des Super-

entlält davon nichts, ebensowenig die anderen älteren Ordnungen der beiden Sachsen, die Sehling zusammenstellt. Ober den Gemeinen Kasten findet sich Genaueres nur in der Instruktion von 1533 (S. 192 b u.). Allein da ist eine andere Art von Rechnungslegung vorgesehen (S. 193 b ii. d. M.).

1) Sehling S. 52 u. 
intendenten soll die Gemeine die Antwort auf die inquisitorischen Fragen durch einen Ausschuß von zwei bis drei geschickten Männern geben. Allein der wird eben nur für diesen $Z$ weck gebildet ${ }^{1}$ ) und hat nur den $Z$ weck, da $B$ nicht von allen durcheinander geredet werde.

2. Der "repräsentative" Charakter, den Brandenburg S. 201 dem Konsistorium zuspricht, schließt auch nach Brandenburgs Meinung (S. 203 oben) keineswegs aus, daß die vertretende Person oder Körperschaft durch eine andere Macht, also auch die Obrigkeit, den Landesherrn, gesetzt wird. Wenn es also im Lätaregutachten S. 144 u. d. M. heißt, das Konsistorium solle „anstatt der ganzen christlichen Kirche dieser Lande sein und das oberste Amt der Schlüssel tragen", oder wenn die drei Superintendenten in ihrem Gutachten (II) ${ }^{2}$ ) das Konsistorium wie den Superintendenten mit seinem Bezirkssenat die allgemeine Kirche repräsentieren und daher ihre richterliche Macht aus der aller andern Kirchen stammen lassen, so ist das nur das Mittel, um die Einrichtung der beiden Gerichte mit Matth. 18, 15-17 und ähnlichen immer wieder verwendeten Beweisstellen in Einklang zu bringen, d. h. sie als spezifisch kirchliche Gerichte zu fordern und von der weltlichen Gerichtsbarkeit zu sondern. Denn während jene geistlichen Gerichte im Namen der ganzen Kirche richten, stellt die weltliche Obrigkeit mit ihren Gerichten nur eine pars ecclesiae dar, der diese Seite nicht überantwortet ist. ${ }^{3}$ ) Dem widerspricht dann im Sinn der Verfasser nicht, daß die Richter, Superintendenten und Konsistorialen, vom Landesherrn ernannt werden: wenn nur - so wird man ihre Absicht vielleicht noch ergänzen dïrfen die "Obrigkeit niedern Stands“4), d. h. der Adel, dabei nicht mitzusprechen hat. Ich glaube also, daß die Ernennung der Konsistorialen durch den Landesherrn für die Konferenz einfach selbstverständlich war, während die Wahl der Superintendenten als der städtischen Pfarrer wie überall, wo das Patronat zu Recht besteht, dem städtischen Rat zukommt ${ }^{5}$ ) und für die übrigen Mitglieder des Bezirksgerichts überhaupt keine Bestimmung getroffen wird.

3. Auch die Art, wie das Lätaregutachten die Visitation eingerichtet wünscht, hat nicht die Bedeutung, die Brandenburg

1) Sehling S. 129 oben.

2) Brandenburg S. 223 bei Nota Abs. 2 (s. o. S. 25 f.).

3) Brandenburg S. 224 u. d. M.

4) Ebd.

s) Sehling S. 123 u. d. M. 
30 Karl Müller, Die Anfänge der Konsistorialverfassung etc.

S.203f. ihr gibt, daß derselbe Geist der Unabhängigkeit von jeder staatlichen Fessel darin erscheine. Man darf diese Visitation, die das Sendgericht nachbilden und lediglich die kirchliche Zucht handhaben soll, nicht verwechseln mit den Visitationen, die die neuen kirchlichen Zustände in ihrem ganzen Umfang begründen und kontrcllieren sollen. Auch wird z. B. bei den Visitationen des Landes, das die straffste landesherrliche Kirchenregierung hat, Württembergs, die eigentlich kirchliche Seite der Arbeit ganz ebenso lediglich durch Geistliche, die Superintendenten, gehandhabt: sie sind dabei aber landesherrliche Organe kirchlicher Art. Und gerade so ist m. E. der Gedanke der Lätarekonferenz, die sich überdies dabei ganz an das Vorbild des Wittenberger Entwurfs von 1542 anlehnt, in dem ja auch das Konsistorium als landesherrliches Kirchengericht besteht. Der Unterschied ist also nur der, daß das Lätaregutachten unter dem Konsistorium noch geistliche $\mathrm{Be}$ zirksgerichte einführen möchte. 